\title{
Besov regularity for the generalized local time of the indefinite Skorohod integral ${ }^{\text {और }}$
}

\author{
Zongxia Liang \\ Department of Mathematical Sciences, Tsinghua University, Beijing 100084, People's Republic of China
}

Received 27 April 2005; received in revised form 3 November 2005; accepted 13 January 2006

Available online 11 July 2006

\begin{abstract}
Let $X_{t}=\int_{0}^{t} u_{s} \mathrm{~d} W_{s}(t \in[0,1])$ be the indefinite Skorohod integral on the canonical probability space $(\Omega, \mathcal{F}, \mathbf{P})$, and let $L_{t}(x)$ $(t \in[0,1], x \in \mathfrak{R})$ be its the generalized local time introduced by Tudor in [C.A. Tudor, Martingale-type stochastic calculus for anticipating integral processes, Bernoulli 10 (2004) 313-325]. We prove that the generalized local time, as function of $x$, has the same Besov regularity as the Brownian motion, as function of $t$, under some conditions imposed on the anticipating integrand $u$. (C) 2006 Elsevier Masson SAS. All rights reserved.
\end{abstract}

\section{Résumé}

Soit $X_{t}=\int_{0}^{t} u_{S} \mathrm{~d} W_{s}(t \in[0,1])$ la primitive de Skorohod sur l'espace de probabilité canonique $(\Omega, \mathcal{F}, \mathbf{P})$, et soit $L_{t}(x)$ $(t \in[0,1], x \in \mathfrak{R})$ le temps local généralisé associé introduit par Tudor dans [C.A. Tudor, Martingale-type stochastic calculus for anticipating integral processes, Bernoulli 10 (2004) 313-325]. On montre que sous certaines conditions sur la fonction à intégrer $u$, le temps local généralisé considéré comme fonction de $x$, a la même régularité Besov que le mouvement Brownien considéré comme fonction de $t$.

(C) 2006 Elsevier Masson SAS. All rights reserved.

MSC: primary 60H07, 60H30; secondary 60G15

Keywords: Besov spaces; Generalized local times; Malliavin calculus; Skorohod integral; Itô-Skorohod integral

\section{Introduction and main results}

Let $\left\{W_{t}, t \in[0,1]\right\}$ be one $\mathfrak{R}$-valued Brownian motion on the canonical probability space $(\Omega, \mathcal{F}, \mathbf{P})$, and let $X_{t}=\int_{0}^{t} u_{s} \mathrm{~d} W_{s}(t \in[0,1])$ be the indefinite Skorohod integral. To give a more probabilistic approach for studying the anticipating stochastic integral $X_{t}$, Tudor recently introduced in [14] the Itô-Skorohod integral which has more properties (somewhat like martingale property) than the Skorohod integral, then he used the Itô-Skorohod integral to define the generalized local time of $X_{t}$ by

$$
\left(X_{t}-x\right)^{+}=(-x)^{+}+\int_{0}^{t} I_{[x,+\infty)}\left(X_{t}^{s}\right) \mathbf{E}\left[v_{s} \mid \mathcal{F}_{[s, t]^{c}}\right] \mathrm{d} W_{s}+\frac{1}{2} L_{t}(x)
$$

\footnotetext{
t) This work is supported by NSFC and SRF for ROCS, SEM.

E-mail address: zliang@math.tsinghua.edu.cn (Z. Liang).
} 
where $v_{s}=u_{s}+\int_{0}^{s} D_{s} u_{t} \mathrm{~d} W_{t}, s \in[0,1], X_{t}^{s}=\int_{0}^{s} \mathbf{E}\left[v_{r} \mid \mathcal{F}_{[r, t]^{c}}\right] \mathrm{d} W_{r}(s \leqslant t)$ is the Itô-Skorohod integral of $v$, and $D$

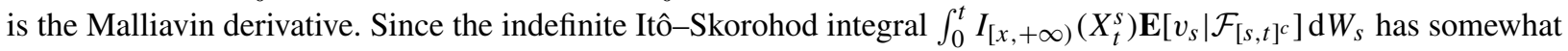
of martingale property, we can avoid the unpleasant fact: the function $I_{[a,+\infty)}\left(X_{S}\right)$ is not Malliavin differentiable (see Imkeller, Malliavin, Nualart $[8,11,12]$ ) when we do estimates of the indefinite Skorohod integral. Therefore there exists a possibility to study fractional smoothness (Besov regularity) of the generalized local time $L_{t}(x)$. If the integrand $u$ is adapted, then the $L_{t}(x)$ is the usual local time of the indefinite Ito stochastic integral, and there is a large number of results on the local time and its continuity (see [6] and references therein). However, there is little result on the local time (see $[8,7,14,16]$ and references therein), and the method used in [6] would not work here when $u$ is non-adapted.

Recently, Boufoussi and Roynette in [4], and Liang in [10] proved that the local times of Brownian motion $W$ and the indefinite Itô stochastic integral process, as functions of $x$, have the same Besov regularity as the Brownian motion as function of $t$ if we impose some conditions on the adapted process $u$. The aim of this work is to extend the results to the indefinite Skorohod integral ( $u$ is non-adapted) by using the technique of the Itô-Skorohod integral and Malliavin calculus, the recent results in Ciesielski, Kerkyacharian, Roynette [5] stating that the Besov spaces are isomorphic to some Banach sequence spaces, the well known results due to Barlow and Yor [1] and the approach used by Nualart, Tudor, Ouknine, Lakhel, Liang and other authors in $[3,9,10,13,14]$ and references therein. More precisely, we will prove the following.

Theorem 1.1. Assume that the anticipating integrand $u$ satisfies the following.

(C1) $u \in \mathbb{L}^{k, p}$ and for $k \geqslant 3$ and any $p \geqslant 2$,

$$
\int_{0}^{1} \mathbf{E}\left|u_{s}\right|^{p} \mathrm{~d} s+\int_{0}^{1} \int_{0}^{1} \mathbf{E}\left|D_{s} u_{r}\right|^{p} \mathrm{~d} s \mathrm{~d} r+\iint_{0}^{1} \int_{0}^{1} \int_{0}^{1} \mathbf{E}\left|D_{\alpha} D_{s} u_{r}\right|^{p} \mathrm{~d} \alpha \mathrm{d} s<+\infty .
$$

(1) If $u$ satisfies the condition (C1), then for every $t>0$ and $p \geqslant 1$ the path $x \rightarrow L_{t}(x)$ almost surely belongs to the Besov space $\mathcal{B}_{p, \infty}^{\frac{1}{2}}$.

(2) If $u$ satisfies the condition $(\mathrm{C} 1)$ and the following condition

(C2) $\mathbf{E}\left[\left(u_{s}+\int_{0}^{s} D_{s} u_{r} \mathrm{~d} W_{r}\right) \mid \mathcal{F}_{s}\right] \neq 0$ a.s. $(s, \omega), \mathrm{d} s \times \mathbf{P}$ on $[0, t] \times \Omega$ for every $t>0$.

Then the path $x \rightarrow L_{t}(x)$ almost surely does not belong to $\mathcal{B}_{p, \infty}^{\frac{1}{2}, 0}$ for every $t>0$ and $p \geqslant 1$.

Where the Besov space $\mathcal{B}_{p, \infty}^{\alpha, 0}$ is a closed separable subspace of $\mathcal{B}_{p, \infty}^{\alpha}, D$ is the Malliavin derivative (see Sections 2 and 3 below) and $\mathcal{F}_{s}$ denotes the $\sigma$-algebra generated by the increments of the Wiener process $W$ on $[0,1] \backslash[s, t]$.

Using the well-known inclusions on Besov spaces (see [2,15]): $\mathcal{B}_{p, q}^{\alpha+\varepsilon} \subset \mathcal{B}_{p, q}^{\alpha}(\varepsilon>0, p, q \in[1, \infty])$ and $\mathcal{B}_{p, q_{1}}^{\alpha} \subset$ $\mathcal{B}_{p, q_{2}}^{\alpha}\left(1 \leqslant q_{1} \leqslant q_{2}, p \in[1, \infty]\right)$, the following result is a straightforward consequence of Theorem 1.1. It states that the generalized local time, as function of $x$, has the same Besov regularity as the Brownian motion as function of $t$.

Corollary 1.1. Assume that the anticipating integrand $u$ satisfies the same conditions as in Theorem 1.1, then we have the following.

(1) The path $x \rightarrow L_{t}(x)$ almost surely belongs to the Besov space $\mathcal{B}_{p, q}^{\alpha}$ for $\alpha<\frac{1}{2}, p, q \in[1, \infty]$.

(2) The path $x \rightarrow L_{t}(x)$ almost surely does not belong to the Besov space $\mathcal{B}_{p, q}^{\alpha}$ for $\alpha>\frac{1}{2}, p, q \in[1, \infty]$.

(3) The path $x \rightarrow L_{t}(x)$ almost surely does not belong to the Besov space $\mathcal{B}_{p, q}^{\frac{1}{2}}$ for $p \in[1, \infty]$ and $q \in[1, \infty)$.

Example 1.1. Let $u_{s}=W_{t} W_{s}$ for any $0 \leqslant s \leqslant t \leqslant 1$, then $D_{s} u_{r}=W_{r} \cdot I_{[0, t]}(s)+W_{t} \cdot I_{[0, r]}(s)$. Moreover, $\mathbf{E}\left[2\left(u_{s}+\right.\right.$ $\left.\left.\int_{0}^{s} D_{s} u_{r} \mathrm{~d} W_{r}\right) \mid \mathcal{F}_{s}\right]=3 W_{s}^{2}-s \neq 0$ a.s. $(s, \omega), \mathrm{d} s \times \mathbf{P}$ on $[0, t] \times \Omega$ for every $t>0$, i.e., the stochastic process $u$ satisfies the condition (C2) above. 
The paper is organized as follows. Sections 2 and 3 contain some preliminaries on Malliavin calculus and the Besov spaces, and in Section 4 we study the Besov regularity of $L_{t}(x)$ with respect to $x$.

\section{Malliavin calculus}

In this section we will give some elements of the Malliavin calculus which mainly come from $[11,12,14]$ and will be useful for this work. Let $W_{t}(t \in[0,1])$ be the standard Wiener process on the canonical Wiener space $(\Omega, \mathcal{F}, \mathbf{P})$, and let $\mathcal{F}_{t}(t \in[0,1])$ be the natural filtration generated by $W$ and $\mathcal{F}_{[s, t]^{c}}$ denote the $\sigma$-algebra generated by the increments of the Wiener process $W$ on $[0,1] \backslash[s, t]$. By $\mathcal{S}$ we denote the set of smooth random variables $(\Omega, \mathcal{F}, \mathbf{P})$, that is, every $F \in \mathcal{S}$ has the form

$$
F=f\left(W_{t_{1}}, \ldots, W_{t_{n}}\right)
$$

with $t_{1}, \ldots, t_{n} \in[0,1]$ and $f \in C_{b}^{\infty}\left(\Re^{n}\right)$ ( $f$ is infinitely continuous differentiable function on $\Re^{n}$ such that $f$ and all of its derivatives are bounded). The Malliavin derivative is defined on $\mathcal{S}$ by

$$
D_{t} F=\sum_{i=1}^{n} \frac{\partial f}{\partial x_{i}}\left(W_{t_{1}}, \ldots, W_{t_{n}}\right) I_{\left[0, t_{i}\right]}(t), \quad t \in[0,1]
$$

if $F$ has the form (2.1). The operator $D$ from $L^{2}(\Omega)$ to $L^{2}([0,1] \times \Omega)$ is closable and its domain (denoted by $\mathbb{L}^{1,2}$ ) is the closure of $\mathcal{S}$ with respect to the norm

$$
\|F\|_{1,2}^{2}=\mathbf{E}|F|^{2}+\mathbf{E}\|D F\|_{L^{2}([0,1])}^{2} .
$$

More generally, we can introduce, for $k$ integer and $p$ real, the space $\mathbb{L}^{k, p}$ of Malliavin differentiable random variables as the closure of $\mathcal{S}$ with respect to the seminorm

$$
\|F\|_{k, p}^{p}=\mathbf{E}|F|^{p}+\sum_{j=1}^{k} \mathbf{E}\left\|D^{(j)} F\right\|_{L^{2}\left([0,1]^{j}\right)}^{p}
$$

where $D^{(j)}$ denotes the $j$ th iterated derivative. Note that if $F$ is $\mathcal{F}_{A}$-measurable ( $A$ being a Borel subset of $\mathfrak{R}$ ), then $D F=0$ on $A^{c} \times \Omega$. The adjoint of $D$, denoted by $\delta$, is defined on the domain

$$
\operatorname{Dom}(\delta)=\left\{u \in L^{2}([0,1] \times \Omega):\left|\mathbf{E} \int_{0}^{1} u_{s} D_{s} F \mathrm{~d} s\right| \leqslant C\|F\|_{L^{2}(\Omega)}\right\}
$$

and it is given by the duality relationship

$$
\mathbf{E}(F \delta(u))=\mathbf{E} \int_{0}^{1} u_{s} D_{s} F \mathrm{~d} s, \quad u \in \operatorname{Dom}(\delta), F \in \mathcal{S} .
$$

We will use the notation $\delta(u)=\int_{0}^{1} u_{s} \mathrm{~d} W_{s}$ and we call $\delta(u)$ the Skorohod integral of $u$. If $\mathbb{H}$ is a real separable Hilbert space, $\mathbb{L}^{k, p}(\mathbb{H})$ will denote the corresponding Banach space of $\mathbb{H}$-valued random variables. It holds that if the process $u \in \mathbb{L}^{1,2}$, then $u I_{[0, t]}$ belongs to $\operatorname{Dom}(\delta)$ for every $t$ and we can consider the indefinite Skorohod integral $X_{t}=\delta\left(u I_{[0, t]}\right)=\int_{0}^{t} u_{s} \mathrm{~d} W_{s}$. Therefore, similar to that of [14], we define, for $k \geqslant 1$ and $p \geqslant 2$, the sets of processes

$$
\mathcal{M}^{k, p}=\left\{X=\left(X_{t}\right)_{t \in[0,1]}, X_{t}=\int_{0}^{t} u_{s} \mathrm{~d} W_{s}, u \in \mathbb{L}^{k, p}\right\}
$$

and

$$
\mathcal{N}^{k, p}=\left\{Y=\left(Y_{t}\right)_{t \in[0,1]}, Y_{t}=\int_{0}^{t} \mathbf{E}\left[v_{s} \mid \mathcal{F}_{[s, t]^{c}}\right] \mathrm{d} W_{s}, v \in \mathbb{L}^{k, p}\right\} .
$$


We will call the elements of the $\mathcal{N}^{k, p}$ as Itô-Skorohod integral processes. Now we list some results here, which can be found in [14], and they will be used in Section 4.

Lemma 2.1. (see Proposition 1 in [14]) Let $X_{t}=\int_{0}^{t} u_{s} \mathrm{~d} W_{s}$ and $u$ belong to $\mathbb{L}^{k, p}$ with $k \geqslant 3$, $p>2$. Then there exists a unique process $v \in \mathbb{L}^{k-2, p}$ such that $X_{t}=\int_{0}^{t} \mathbf{E}\left[v_{s} \mid \mathcal{F}_{[s, t]^{c}}\right] \mathrm{d} W_{s}$ for every $t \in[0,1]$. Moreover, $v .=u .+\int_{0}^{s} D . u_{s} \mathrm{~d} W_{s}$.

Lemma 2.2. (see Propositions 5 and 6 in [14]) Let $Y_{t}^{\lambda}=\int_{0}^{\lambda} \mathbf{E}\left[u_{s} \mid \mathcal{F}_{[s, t]^{c}}\right] \mathrm{d} W_{s}$ for $\lambda \leqslant t$ and $Y_{t}=\int_{0}^{t} \mathbf{E}\left[u_{s} \mid \mathcal{F}_{\left.[s, t]^{c}\right] \mathrm{d} W_{s}}\right.$ with $u \in L^{2}([0,1] \times \Omega)$ and let $\Phi$ be a Borel function. Then there exists a non-negative process $L_{t}(x)(t \in[0,1]$, $x \in \mathfrak{R}$ ) such that (Tanaka formula)

$$
\left(Y_{t}-x\right)^{+}=(-x)^{+}+\int_{0}^{t} I_{[x,+\infty)}\left(Y_{t}^{s}\right) \mathbf{E}\left[u_{s} \mid \mathcal{F}_{\left.[s, t]^{c}\right]} \mathrm{d} W_{s}+\frac{1}{2} L_{t}(x),\right.
$$

(occupation time formula)

$$
\int_{0}^{t} \Phi\left(Y_{t}^{s}\right)\left(\mathbf{E}\left[u_{s} \mid \mathcal{F}_{[s, t]}\right]\right)^{2} \mathrm{~d} s=\int_{-\infty}^{\infty} \Phi(x) L_{t}(x) \mathrm{d} x
$$

and

$$
\lim _{\lambda \uparrow t} L_{t}^{\lambda}(x)=L_{t}(x) \quad \text { almost surely and in } L^{2} .
$$

Where $L_{t}^{\lambda}(x)$ is the local time of $Y_{t}^{\lambda}(\lambda \in[0, t])$, that is, it satisfies the following.

$$
\left(Y_{t}^{\lambda}-x\right)^{+}=(-x)^{+}+\int_{0}^{\lambda} I_{[x,+\infty)}\left(Y_{t}^{s}\right) \mathbf{E}\left[u_{s} \mid \mathcal{F}_{[s, t]^{c}}\right] \mathrm{d} W_{s}+\frac{1}{2} L_{t}^{\lambda}(x),
$$

(occupation time formula)

$$
\int_{0}^{\lambda} \Phi\left(Y_{t}^{s}\right)\left(\mathbf{E}\left[u_{s} \mid \mathcal{F}_{[s, t]}\right]\right)^{2} \mathrm{~d} s=\int_{-\infty}^{\infty} \Phi(x) L_{t}^{\lambda}(x) \mathrm{d} x
$$

and

$$
L_{t}^{\lambda}(x)=\lim _{\varepsilon \rightarrow 0} \frac{1}{2 \varepsilon} \int_{0}^{\lambda} I_{(x-\varepsilon, x+\varepsilon)}\left(Y_{t}^{s}\right)\left(\mathbf{E}\left[u_{s} \mid \mathcal{F}_{[s, t]}\right]\right)^{2} \mathrm{~d} s .
$$

If $u \in \mathbb{L}^{k, p}(k \geqslant 3, p>2)$ and $X_{t}=\int_{0}^{t} u_{s} \mathrm{~d} W_{s}$, then $X_{t}=\int_{0}^{t} \mathbf{E}\left[v_{s} \mid \mathcal{F}_{[s, t]^{c}}\right] \mathrm{d} W_{s}$ by Lemma 2.1. By using Lemma 2.2 we can determine a non-negative process $L_{t}^{X}(x)$ such that (2.2) holds with $v .=u .+\int_{0}^{*} D . u_{s} \mathrm{~d} W_{s}$ instead of $u$. We call the process $L_{t}^{X}$ as the generalized local time of the indefinite Skorohod integral $X_{t}$. We still denote it by $L_{t}(x)$ for simplicity in what follows. We will study its Besov regularity in Section 4 . Moreover, we will use the following Meyer's inequalities.

Lemma 2.3. (see Theorem 1.5.1 in [12]) Let $u \in \mathbb{L}^{1, p}$ with $p \geqslant 2$. Then

$$
\mathbf{E}|\delta(u)|^{p} \leqslant\|u\|_{1, p}^{p} .
$$

\section{Besov spaces}

In this section we will collect some facts about the Besov Spaces which will be used later. Let $f:[0,1] \rightarrow \Re$ be a measurable function, and let $1 \geqslant \alpha>0,1 \leqslant p, q \leqslant \infty . L^{p}([0,1] ; \mathfrak{R})(1 \leqslant p<+\infty)$ denotes the space of Lebesgue 
integrable $\mathfrak{R}$-valued functions defined on $I=[0,1]$ with exponent $p .\|\cdot\|_{p}$ denotes the usual $L^{p}([0,1] ; \mathfrak{R})$-norm. Define the modulus $\omega_{p}(f, t)$ of smoothness in $L^{p}([0,1] ; \Re)$ by

$$
\omega_{p}(f, t)=\sup _{0 \leqslant h \leqslant t}\left\|(f(\cdot+h)-f(\cdot)) I_{[0,1-h]}(\cdot)\right\|_{p} .
$$

Let

$$
\|f\|_{\alpha, p, q}= \begin{cases}\|f\|_{p}+\left(\int_{0}^{1}\left(\frac{1}{t^{\alpha}} \omega_{p}(f, t)\right)^{q} \frac{\mathrm{d} t}{t}\right)^{\frac{1}{q}} & \text { if } q<+\infty, \\ \|f\|_{p}+\sup _{0 \leqslant t \leqslant 1} \frac{\omega_{p}(f, t)}{t^{\alpha}} & \text { if } q=+\infty .\end{cases}
$$

We define the Besov space of parameters $\alpha, p, q$ denoted by $\mathcal{B}_{p, q}^{\alpha}$ as the Banach space of functions $f$ such that $\|f\|_{\alpha, p, q}<+\infty$, endowed with this norm. If $q=\infty$, we denote the non-separable Banach space by $\mathcal{B}_{p, \infty}^{\alpha} \cdot \mathcal{B}_{p, \infty}^{\alpha, 0}$ denotes the closed separable subspace of $\mathcal{B}_{p, \infty}^{\alpha}$ formed with the functions of $\mathcal{B}_{p, \infty}^{\alpha}$ satisfying $\omega_{p}(f, t)=\mathrm{o}\left(t^{\alpha}\right)(t \downarrow 0)$.

Let $\chi_{1} \equiv 1, \chi_{j k}=2^{\frac{j}{2}} I_{\left[\frac{k-1}{2^{j}}, \frac{2 k-1}{2^{j+1}}\right)}-2^{\frac{j}{2}} I_{\left[\frac{2 k-1}{2^{j+1}}, \frac{k}{2^{j}}\right)}\left(j=0,1, \ldots ; k=1, \ldots, 2^{j}\right)$ be the complete orthogonal Haar basis in $L^{2}([0,1] ; \Re)$ and $\varphi_{0} \equiv 1, \varphi_{1}(x)=x, \varphi_{j k}(x)=\int_{0}^{x} \chi_{j k}(s) \mathrm{d} s$ the Schauder basis on [0,1]. It is well known that for all continuous functions $f$ on $[0,1]$ we have the following decomposition:

$$
f(x)=f_{0}+f_{1} \varphi_{1}(x)+\sum_{j, k} f_{j k} \varphi_{j k}(x)
$$

with $f_{0}=f(0), f_{1}=f(1)-f(0)$ and

$$
f_{j k}=22^{\frac{j}{2}}\left[f\left(\frac{2 k-1}{2^{j+1}}\right)-\frac{1}{2}\left(f\left(\frac{2 k}{2^{j+1}}\right)+f\left(\frac{2 k-2}{2^{j+1}}\right)\right)\right] .
$$

It was proved in [5] (see Theorem III.6) that for $\frac{1}{p}<\alpha<1+\frac{1}{p}$ we have the following equivalence of norms:

$$
\|f\|_{\alpha, p, q} \sim \begin{cases}\left(\sum_{j} 2^{-j q\left(\frac{1}{2}-\alpha+\frac{1}{p}\right)}\left(\sum_{k}\left|f_{j k}\right|^{p}\right)^{\frac{q}{p}}\right)^{\frac{1}{q}}+\left(\left|f_{0}\right|^{q}+\left|f_{1}\right|^{q}\right)^{\frac{1}{q}} & \text { if } q<+\infty \\ \sup \left\{\left|f_{0}\right|,\left|f_{1}\right|, \sup _{j} 2^{-j\left(\frac{1}{2}-\alpha+\frac{1}{p}\right)}\left(\sum_{k}\left|f_{j k}\right|^{p}\right)^{\frac{1}{p}}\right\} & \text { if } q=+\infty .\end{cases}
$$

It has also been shown that a function $f$ belongs to $\mathcal{B}_{p, \infty}^{\alpha, 0}$ if and only if

$$
\lim _{j \rightarrow+\infty} \sup _{j} 2^{-j\left(\frac{1}{2}-\alpha+\frac{1}{p}\right)}\left(\sum_{k}\left|f_{j k}\right|^{p}\right)^{\frac{1}{p}}=0 .
$$

\section{Besov regularity of the generalized local time $L_{t}(x)$}

In this section we will study the Besov regularity of the generalized local time $L_{t}(x)$ of $X_{t}=\int_{0}^{t} u_{s} \mathrm{~d} W_{s}$ defined in Section 2. Assume that the anticipating integrand $u$ satisfies the conditions in Theorem 1.1, and let $t=1$ for convenience, and through the paper the $C(p, q)$ will denote a universal constant depending only on $p$ and $q$. Now we give

Proof of Theorem 1.1. By Lemma 2.1, the Tanaka formula (2.2) and $(x-a)^{+}=\int_{0}^{x} I_{[a, \infty)}(s) \mathrm{d} s$ we have the following decomposition of $L_{1}(x)$,

$$
L_{1}(x)=L_{1}(0)+\left(L_{1}(1)-L_{1}(0)\right) \varphi_{1}(x)+\sum_{j k}\left(L_{1}\right)_{j k} \varphi_{j k}(x), \quad x \in[0,1]
$$

with

$$
\left(L_{1}\right)_{j k}=2 \varphi_{j k}\left(X_{1}\right)-2 \int_{0}^{1} \chi_{j k}\left(X_{1}^{s}\right) \mathbf{E}\left[v_{s} \mid \mathcal{F}_{s}\right] \mathrm{d} W_{s}
$$


where $X_{1}^{t}=\int_{0}^{t} \mathbf{E}\left[v_{s} \mid \mathcal{F}_{s}\right] \mathrm{d} W_{s}(t \in[0,1])$. If we let $M_{j k}(t)=\int_{0}^{t} \chi_{j k}\left(X_{1}^{s}\right) \mathbf{E}\left[v_{s} \mid \mathcal{F}_{s}\right] \mathrm{d} W_{s}\left(j=0,1, \ldots ; k=1, \ldots, 2^{j}\right.$, $t \in[0,1])$, then by using the equivalence of norms (3.3) and (3.4) the proof of Theorem 1.1 can be reduced to proving the following

$$
\begin{aligned}
& \liminf _{j \longrightarrow \infty} 2^{-j} \sum_{k}\left|\varphi_{j k}\left(X_{1}\right)-M_{j k}(1)\right|^{p}>0 \quad \text { almost surely, } \\
& \sup _{j} 2^{-j} \sum_{k}\left|\varphi_{j k}\left(X_{1}\right)-M_{j k}(1)\right|^{p}<+\infty
\end{aligned}
$$

for any $p>2$.

Because for $p>0$

$$
2^{-j} \sum_{k}\left|\varphi_{j k}\left(X_{1}\right)\right|^{p} \leqslant 2^{-\frac{j p}{2}} \longrightarrow 0 \quad \text { as } j \longrightarrow \infty,
$$

the proofs of (4.3) and (4.4) can be reduced to proving the following

$$
\begin{aligned}
& \liminf _{j \longrightarrow \infty} 2^{-j} \sum_{k}\left|M_{j k}(1)\right|^{p}>0 \quad \text { almost surely, } \\
& \sup _{j} 2^{-j} \sum_{k}\left|M_{j k}(1)\right|^{p}<+\infty \quad \text { almost surely }
\end{aligned}
$$

for any $p>2$. Before proving the (4.5) and (4.6) we need to establish the following lemmas.

Lemma 4.1. Assume the anticipating integrand $u$ satisfies the condition $(\mathrm{C} 1)$ in Theorem 1.1. Let $L_{1}^{1, *}=\sup _{x} L_{1}^{1}(x)$. Then there is a universal constant $C(p)$ such that

$$
\mathbf{E}\left(L_{1}^{1, *}\right)^{p} \leqslant C(p)\left[\int_{0}^{1} \mathbf{E}\left|u_{s}\right|^{p} \mathrm{~d} s+\iint_{0}^{1} \int_{0}^{1} \mathbf{E}\left|D_{s} u_{r}\right|^{p} \mathrm{~d} s \mathrm{~d} r+\iint_{0}^{1} \int_{0}^{1} \mathbf{E}\left|D_{\alpha} D_{s} u_{r}\right|^{p} \mathrm{~d} \alpha \mathrm{d} s \mathrm{~d} r\right]
$$

for $p \geqslant 2$.

Proof. By the results on local times inequalities proved by Barlow and Yor in [1] (see (III ${ }_{\gamma}$ ) of Corollary 5.2.2), the stochastic process $X_{1}^{\lambda}=\int_{0}^{\lambda} \mathbf{E}\left[v_{s} \mid \mathcal{F}_{S}\right] \mathrm{d} W_{S}(\lambda \in[0,1])$ is an $\mathcal{F}_{\lambda}$ - martingale by Lemma 2.2, and the Burkholder-DavisGundy inequalities we have

$$
\begin{aligned}
\mathbf{E}\left(L_{1}^{1, *}\right)^{p} & \leqslant C(p) \mathbf{E}\left\{\int_{0}^{1}\left(\mathbf{E}\left[v_{s} \mid \mathcal{F}_{s}\right]\right)^{2} \mathrm{~d} s\right\}^{\frac{p}{2}} \\
& \leqslant C(p) \int_{0}^{1} \mathbf{E}\left|v_{s}\right|^{p} \mathrm{~d} s \quad \text { (by the Jensen's inequality) } \\
& \leqslant C(p) \int_{0}^{1} \mathbf{E}\left|u_{s}\right|^{p} \mathrm{~d} s+C(p) \int_{0}^{1} \mathbf{E}\left|\int_{0}^{s} D_{s} u_{r} \mathrm{~d} W_{r}\right|^{p} \mathrm{~d} s .
\end{aligned}
$$

On the other hand, by using the Meyer's inequalities (Lemma 2.3) and the Hölder's inequality,

$$
\begin{aligned}
\mathbf{E}\left|\int_{0}^{s} D_{s} u_{r} \mathrm{~d} W_{r}\right|^{p} & =\mathbf{E}\left|\delta\left(I_{[0, s]}(\cdot) D_{s} u .\right)\right|^{p} \leqslant C(p) \mathbf{E}\left(\int_{0}^{1}\left|D_{s} u_{r}\right|^{2} \mathrm{~d} r\right)^{\frac{p}{2}}+C(p) \mathbf{E}\left(\iint_{0}^{1}\left|D_{\alpha} D_{s} u_{r}\right|^{2} \mathrm{~d} r \mathrm{~d} \alpha\right)^{\frac{p}{2}} \\
& \leqslant C(p) \mathbf{E} \int_{0}^{1}\left|D_{s} u_{r}\right|^{p} \mathrm{~d} r+C(p) \mathbf{E} \int_{0}^{1} \int_{0}^{1}\left|D_{\alpha} D_{s} u_{r}\right|^{p} \mathrm{~d} r \mathrm{~d} \alpha
\end{aligned}
$$

Thus (4.8) and (4.9) imply the proof. 
Lemma 4.2. Assume that the anticipating integrand $u$ satisfies the condition $(\mathrm{C} 1)$ in Theorem 1.1. Then we have

$$
\begin{aligned}
& \lim _{j \rightarrow \infty} 2^{-j} \sum_{k} \int_{0}^{1} M_{j k}^{p}(s) \chi_{j k}\left(X_{1}^{s}\right) \mathbf{E}\left[v_{s} \mid \mathcal{F}_{s}\right] \mathrm{d} W_{s}=0, \\
& \lim _{j \rightarrow \infty} 2^{-j} \sum_{k} \int_{0}^{1} M_{j k}^{p}(s)\left(\int_{0}^{s} \chi_{j k}^{2}\left(X_{1}^{\alpha}\right)\left(\mathbf{E}\left[v_{\alpha} \mid \mathcal{F}_{\alpha}\right]\right)^{2} \mathrm{~d} \alpha\right) \chi_{j k}\left(X_{1}^{s}\right) \mathbf{E}\left[v_{s} \mid \mathcal{F}_{s}\right] \mathrm{d} W_{s}=0
\end{aligned}
$$

almost surely for $p \geqslant 1$.

Proof. Since the martingales $\int_{0}^{t} M_{j k}^{p}(s) \chi_{j k}\left(X_{1}^{s}\right) \mathbf{E}\left[v_{s} \mid \mathcal{F}_{s}\right] \mathrm{d} W_{s}\left(k=1, \ldots, 2^{j}, 0 \leqslant t \leqslant 1\right)$ are orthogonal, we deduce from the occupation formula (2.3) that

$$
\begin{aligned}
\mathbf{E}\left(\sum_{k} \int_{0}^{1} M_{j k}^{p}(s) \chi_{j k}\left(X_{1}^{s}\right) \mathbf{E}\left[v_{s} \mid \mathcal{F}_{s}\right] \mathrm{d} W_{s}\right)^{2} & =\sum_{k} \mathbf{E}\left(\int_{0}^{1} M_{j k}^{p}(s) \chi_{j k}\left(X_{1}^{s}\right) \mathbf{E}\left[v_{s} \mid \mathcal{F}_{s}\right] \mathrm{d} W_{s}\right)^{2} \\
& =\sum_{k} \mathbf{E}\left(\int_{0}^{1} M_{j k}^{2 p}(s) \chi_{j k}^{2}\left(X_{1}^{s}\right)\left(\mathbf{E}\left[v_{s} \mid \mathcal{F}_{s}\right]\right)^{2} \mathrm{~d} s\right) \\
& \leqslant \sum_{k} \mathbf{E}\left(M_{j, k}^{*}{ }^{2 p} \int_{0}^{1} \chi_{j k}^{2}\left(X_{1}^{s}\right)\left(\mathbf{E}\left[v_{s} \mid \mathcal{F}_{s}\right]\right)^{2} \mathrm{~d} s\right) \\
& =\sum_{k} \mathbf{E}\left(M_{j, k}^{*} \int_{\mathfrak{R}}^{2 p} \chi_{j k}^{2}(x) L_{1}(x) \mathrm{d} x\right) \\
& \leqslant \sum_{k} \mathbf{E}\left(M_{j, k}^{*}{ }^{2 p} L_{1}^{1, *} \int_{\mathfrak{R}} \chi_{j k}^{2}(x) \mathrm{d} x\right) \\
& \leqslant \sum_{k} \mathbf{E}\left(M_{j, k}^{*}{ }^{2 p} L_{1}^{1, *}\right) \\
& \leqslant \sum_{k}\left|\mathbf{E}\left(M_{j, k}^{*}{ }^{4 p}\right)\right|^{\frac{1}{2}}\left|\mathbf{E}\left(L_{1}^{1, *}\right)^{2}\right|^{\frac{1}{2}}
\end{aligned}
$$

by the Hölder's inequality, and $L_{1}^{1, *} \geqslant L_{1}^{1}(x) \geqslant \lim _{\lambda \uparrow 1} L_{1}^{\lambda}(x)=L_{1}(x)$ almost surely because $L_{t}^{\lambda}(x)$ is increasing in $\lambda$, where $M_{j, k}^{*}=\sup _{t \in[0,1]}\left|M_{j, k}(t)\right|$.

By the Burkholder-Davis-Gundy inequalities, the occupation formula (2.3), the Hölder's inequality and Lemma 4.1 one has

$$
\begin{aligned}
\mathbf{E}\left(M_{j, k}^{*}{ }^{4 p}\right) & \leqslant C(p) \mathbf{E}\left(\int_{0}^{1} \chi_{j k}^{2}\left(X_{1}^{s}\right)\left(\mathbf{E}\left[v_{s} \mid \mathcal{F}_{s}\right]\right)^{2} \mathrm{~d} s\right)^{2 p} \\
& =C(p) \mathbf{E}\left(\int_{\mathfrak{R}} L_{1}(x) \chi_{j k}^{2}(x) \mathrm{d} x\right)^{2 p} \\
& \leqslant C(p) \mathbf{E}\left(\int_{\mathfrak{R}} \chi_{j k}^{2}(x) \mathrm{d} x\right)^{2 p-1}\left(\int_{\mathfrak{R}}\left(L_{1}(x)\right)^{2 p} \chi_{j k}^{2}(x) \mathrm{d} x\right) \\
& \leqslant C(p) \mathbf{E}\left(L_{1}^{1, *}\right)^{2 p} \\
& \leqslant C(p)\left[\int_{0}^{1} \mathbf{E}\left|u_{s}\right|^{2 p} \mathrm{~d} s+\iint_{0}^{1} \mathbf{E}\left|D_{s} u_{r}\right|^{2 p} \mathrm{~d} s \mathrm{~d} r+\iint_{0}^{1} \int_{0}^{1} \mathbf{E}\left|D_{\alpha} D_{s} u_{r}\right|^{2 p} \mathrm{~d} \alpha \mathrm{d} s \mathrm{~d} r\right]<+\infty .
\end{aligned}
$$


Hence, the inequalities (4.12), (4.13) and Lemma 4.1 yield a constant $C$ such that

$$
\mathbf{E}\left(\sum_{k} \int_{0}^{1} M_{j k}^{p}(s) \chi_{j k}\left(X_{1}^{s}\right) \mathbf{E}\left[v_{s} \mid \mathcal{F}_{s}\right] \mathrm{d} W_{s}\right)^{2} \leqslant 2^{j} C<\infty .
$$

Therefore we know from the last inequality that for any $\varepsilon>0$

$$
\sum_{j=1}^{\infty} \mathbf{P}\left(\left|2^{-j} \sum_{k} \int_{0}^{1} M_{j k}^{p}(s) \chi_{j k}\left(X_{1}^{s}\right) \mathbf{E}\left[v_{s} \mid \mathcal{F}_{s}\right] \mathrm{d} W_{s}\right| \geqslant \varepsilon\right) \leqslant \frac{1}{\varepsilon^{2}} \sum_{j=1}^{\infty} C 2^{-j}<+\infty .
$$

By the Borel-Cantelli lemma we have

$$
\lim _{j \rightarrow \infty} 2^{-j} \sum_{k} \int_{0}^{1} M_{j k}^{p}(s) \chi_{j k}\left(X_{1}^{s}\right) \mathbf{E}\left[v_{s} \mid \mathcal{F}_{s}\right] \mathrm{d} W_{s}=0, \quad \text { almost surely. }
$$

Noting that $\int_{0}^{s} \chi_{j k}^{2}\left(X_{1}^{\alpha}\right)\left(\mathbf{E}\left[v_{\alpha} \mid \mathcal{F}_{\alpha}\right]\right)^{2} \mathrm{~d} \alpha=\int_{\mathfrak{R}} L_{s}^{\alpha}(x) \chi_{j k}^{2}(x) \mathrm{d} x \leqslant L_{1}^{1, *}$, we can prove (4.11) as the same way as in proving (4.10). Thus the proof of Lemma 4.2 is complete.

Lemma 4.3. Assume that the anticipating integrand $u$ satisfies the condition (C1). Then we have

$$
\sup _{j} 2^{-j} \sum_{k} M_{j k}^{2}(1)<+\infty
$$

almost surely.

Proof. By the Itô's formula we have

$$
\begin{aligned}
M_{j k}^{2}(1) & =2 \int_{0}^{1} M_{j k}(s) \chi_{j k}\left(X_{1}^{s}\right) \mathbf{E}\left[v_{s} \mid \mathcal{F}_{s}\right] \mathrm{d} W_{s}+\int_{0}^{1} \chi_{j k}^{2}\left(X_{1}^{s}\right)\left(\mathbf{E}\left[v_{s} \mid \mathcal{F}_{s}\right]\right)^{2} \mathrm{~d} s \\
& \equiv 2 I_{j k}^{1}+I_{j k}^{2} .
\end{aligned}
$$

Using Lemma 4.2,

$$
\lim _{j \rightarrow \infty} 2^{-j} \sum_{k} I_{j k}^{1}=0, \quad \text { almost surely. }
$$

Since

$$
2^{-j} \sum_{k} I_{j k}^{2}=\int_{0}^{1} I_{[0,1]}\left(X_{1}^{s}\right)\left(\mathbf{E}\left[v_{s} \mid \mathcal{F}_{s}\right]\right)^{2} \mathrm{~d} s=\int_{\mathfrak{R}} I_{[0,1]}(x) L_{1}(x) \mathrm{d} x \leqslant L_{1}^{1, *}<+\infty
$$

by Lemma 4.1 and the condition (C1), the proof is complete by (4.16).

Now we first return to proving (4.5).

By the Hölder's inequality we have

$$
2^{-j} \sum_{k}\left|M_{j k}(1)\right|^{p} \geqslant\left[2^{-j} \sum_{k} M_{j k}^{2}\right]^{\frac{p}{2}} \text {. }
$$

On the other hand, we deduce from the (4.15)-(4.17) and the condition (C2) that

$$
\liminf _{j \rightarrow \infty} 2^{-j} \sum_{k}\left|M_{j k}(1)\right|^{2} \geqslant \int_{0}^{1} I_{[0,1]}\left(X_{1}^{s}\right)\left(\mathbf{E}\left[v_{s} \mid \mathcal{F}_{s}\right]\right)^{2} \mathrm{~d} s>0
$$


almost surely. Thus the last two inequalities imply the (4.5).

Next we return to proving (4.6).

We prove (4.6) by induction on $p$. We assume that the following hypotheses are satisfied

$$
\left\{\begin{array}{l}
\sup _{j} 2^{-j} \sum_{k}\left|M_{j k}(1)\right|^{2 p}<+\infty \quad \text { almost surely, } \\
\sup _{j} 2^{-j} \sum_{k} \int_{0}^{1} M_{j k}^{2 p-2}(s) \chi_{j k}^{2}\left(X_{1}^{s}\right)\left(\mathbf{E}\left[v_{s} \mid \mathcal{F}_{s}\right]\right)^{2} \mathrm{~d} s<+\infty \quad \text { almost surely }
\end{array}\right.
$$

for $p \geqslant 1$.

By Lemma 4.3 this property holds for $p=1$. Now we want to show that the hypotheses (4.19) hold for $p+1$. Applying the Itô formula to the process $\left\{M_{j k}^{2 p+2}(t), t \geqslant 0\right\}$ we have

$$
\begin{aligned}
\left|M_{j k}(1)\right|^{2 p+2}= & 2(p+1) \int_{0}^{1} M_{j k}^{2 p+1}(s) \chi_{j k}\left(X_{1}^{s}\right) \mathbf{E}\left[v_{s} \mid \mathcal{F}_{s}\right] \mathrm{d} W_{s} \\
& +(p+1)(2 p+1) \int_{0}^{1} M_{j k}^{2 p}(s) \chi_{j k}^{2}\left(X_{1}^{s}\right)\left(\mathbf{E}\left[v_{s} \mid \mathcal{F}_{s}\right]\right)^{2} \mathrm{~d} s .
\end{aligned}
$$

Using Lemma 4.2 we have

$$
\lim _{j \rightarrow \infty} 2^{-j} \sum_{k} \int_{0}^{1} M_{j k}^{2 p+1}(s) \chi_{j k}\left(X_{1}^{s}\right) \mathbf{E}\left[v_{s} \mid \mathcal{F}_{s}\right] \mathrm{d} W_{s}=0
$$

almost surely.

By the Itô's formula

$$
\begin{aligned}
\left|M_{j k}(t)\right|^{2 p} & =2 p \int_{0}^{t} M_{j k}^{2 p-1}(s) \chi_{j k}\left(X_{1}^{s}\right) \mathbf{E}\left[v_{s} \mid \mathcal{F}_{s}\right] \mathrm{d} W_{s}+p(2 p-1) \int_{0}^{t} M_{j k}^{2 p-2}(s) \chi_{j k}^{2}\left(X_{1}^{s}\right)\left(\mathbf{E}\left[v_{s} \mid \mathcal{F}_{s}\right]\right)^{2} \mathrm{~d} s \\
& \equiv A_{j k}(t)+B_{j k}(t) .
\end{aligned}
$$

By using the decomposition (4.22) and the integration by parts we have

$$
\begin{aligned}
& 2^{-j} \sum_{k} \int_{0}^{1} M_{j k}^{2 p}(s) \chi_{j k}^{2}\left(X_{1}^{s}\right)\left(\mathbf{E}\left[v_{s} \mid \mathcal{F}_{s}\right]\right)^{2} \mathrm{~d} s \\
& \leqslant 2^{-j}\left|\sum_{k} A_{j k}(1) \int_{0}^{1} \chi_{j k}^{2}\left(X_{1}^{s}\right)\left(\mathbf{E}\left[v_{s} \mid \mathcal{F}_{s}\right]\right)^{2} \mathrm{~d} s\right|+2^{-j}\left|\sum_{k} \int_{0}^{1}\left(\int_{0}^{t} \chi_{j k}^{2}\left(X_{1}^{s}\right)\left(\mathbf{E}\left[v_{s} \mid \mathcal{F}_{s}\right]\right)^{2} \mathrm{~d} s\right) \mathrm{d} A_{j k}(t)\right| \\
& \quad+2^{-j}\left|\sum_{k} B_{j k}(1) \int_{0}^{1} \chi_{j k}^{2}\left(X_{1}^{s}\right)\left(\mathbf{E}\left[v_{s} \mid \mathcal{F}_{s}\right]\right)^{2} \mathrm{~d} s\right| \\
& \quad+2^{-j}\left|\sum_{k} \int_{0}^{1}\left(\int_{0}^{t} \chi_{j k}^{2}\left(X_{1}^{s}\right)\left(\mathbf{E}\left[v_{s} \mid \mathcal{F}_{s}\right]\right)^{2} \mathrm{~d} s\right) M_{j k}^{2 p-2}(t) \chi_{j k}^{2}\left(X_{1}^{t}\right)\left(\mathbf{E}\left[v_{t} \mid \mathcal{F}_{t}\right]\right)^{2} \mathrm{~d} t\right|
\end{aligned}
$$

By Lemma 4.2, the occupation formula (2.3) and $L_{1}^{1, *} \geqslant L_{1}^{1}(x) \geqslant \lim _{\lambda \uparrow 1} L_{1}^{\lambda}(x)=L_{1}(x)$

$$
\begin{aligned}
& \sup _{j} 2^{-j}\left|\sum_{k} A_{j k}(1) \int_{0}^{1} \chi_{j k}^{2}\left(X_{1}^{s}\right)\left(\mathbf{E}\left[v_{s} \mid \mathcal{F}_{s}\right]\right)^{2} \mathrm{~d} s\right| \\
& \leqslant L_{1}^{1, *} \sup _{j} 2^{-j} \sum_{k}\left|\int_{0}^{1} M_{j k}^{2 p-1}(s) \chi_{j k}\left(X_{1}^{s}\right) \mathbf{E}\left[v_{s} \mid \mathcal{F}_{s}\right] \mathrm{d} W_{s}\right|<+\infty
\end{aligned}
$$

almost surely. 
By Lemma 4.2 we have

$$
\sup _{j} 2^{-j}\left|\sum_{k} \int_{0}^{1}\left(\int_{0}^{t} \chi_{j k}^{2}\left(X_{1}^{s}\right)\left(\mathbf{E}\left[v_{s} \mid \mathcal{F}_{s}\right]\right)^{2} \mathrm{~d} s\right) \mathrm{d} A_{j k}(t)\right|<+\infty
$$

almost surely. By the second hypothesis in (4.19), the occupation formula (2.3) and $L_{1}^{1, *} \geqslant L_{1}(x)$ we have

$$
\sup _{j} 2^{-j}\left|\sum_{k} B_{j k}(1) \int_{0}^{1} \chi_{j k}^{2}\left(X_{1}^{s}\right)\left(\mathbf{E}\left[v_{s} \mid \mathcal{F}_{s}\right]\right)^{2} \mathrm{~d} s\right| \leqslant L_{1}^{1, *} \sup _{j}\left|2^{-j} \sum_{k} B_{j k}(1)\right|<+\infty
$$

and

$$
\begin{aligned}
& \sup _{j} 2^{-j}\left|\sum_{k} \int_{0}^{1}\left(\int_{0}^{t} \chi_{j k}^{2}\left(X_{1}^{s}\right)\left(\mathbf{E}\left[v_{s} \mid \mathcal{F}_{s}\right]\right)^{2} \mathrm{~d} s\right) M_{j k}^{2 p-2}(t) \chi_{j k}^{2}\left(X_{1}^{t}\right)\left(\mathbf{E}\left[v_{t} \mid \mathcal{F}_{t}\right]\right)^{2} \mathrm{~d} t\right| \\
& \leqslant L_{1}^{1, *} \sup _{j}\left|2^{-j} \sum_{k} B_{j k}(1)\right|<+\infty
\end{aligned}
$$

almost surely. Thus the inequalities (4.20)-(4.21) and (4.25)-(4.28) imply the proof of Theorem 1.1 is complete.

\section{Acknowledgements}

The author would like to express his deep thanks to the referee for his careful reading the manuscript and his valuable suggestions which make the main result of this paper to be optimal. The author is also very grateful to NSFC for the generous support.

\section{References}

[1] M.T. Barlow, M. Yor, Semimartingale inequalities via the Garsia-Rodemich-Rumsey lemma, and applications to local times, J. Funct. Anal. 49 (1982) 198-229.

[2] J. Bergh, J. Löfström, Interpolation Spaces - An Introduction, Springer-Verlag, World Publishing Corp., 1976, 2003.

[3] A. Berkaoui, Y. Ouknine, Régularité Besov des trajectoires du processus intégral de Skorohod, Bull. Sci. Math. 123 (1999) $643-663$.

[4] B. Boufoussi, B. Roynette, Le temps local brownien appartient p.s. l'espace de Besov $\mathcal{B}_{p, \alpha}^{1 / 2}$, C. R. Acad. Sci. Paris Ser. I Math. 316 (1993) 843-848.

[5] Z. Ciesielski, G. Kerkyacharian, B. Roynette, Quelques espaces fonctionnels associés des processus gaussiens, Studia Math. 107 (1993) 171-204.

[6] D. Geman, J. Horowitz, Occupation densities, Ann. Probab. 8 (1980) 1-67.

[7] P. Imkeller, Occupation densities for stochastic integral processes in the second Wiener chaos, Probab. Theory Related Fields 91 (1992) 1-24.

[8] P. Imkeller, D. Nualart, Integration by parts on Wiener space and the existence of occupation densities, Ann. Probab. 22 (1994) 469-493.

[9] H. Lakhel, Y. Ouknine, C.A. Tudor, Besov regularity for the indefinite Skorohod integral with respect to the fractional Brownian motion: the singular case, Stochastics Stochastics Rep. 74 (2002) 597-615.

[10] Z. Liang, Besov-regularity of local times of some semimartingales, Preprint, 2005.

[11] P. Malliavin, Stochastic Analysis, Grundlehren Math. Wiss. (Fundamental Principles of Mathematical Sciences), vol. 313, Springer-Verlag, Berlin, ISBN 3-540-57024-1, 1997 (xii+343 pp.).

[12] D. Nualart, The Malliavin Calculus and Related Topics, Probab. Appl., Springer-Verlag, New York, ISBN 0-387-94432-X, 1995 (xii+266 pp.).

[13] D. Nualart, Y. Ouknine, Besov regularity of stochastic integrals with respect to the fractional Brownian motion with parameter $H>\frac{1}{2}$, J. Theoret. Probab. 16 (2003) 451-470.

[14] C.A. Tudor, Martingale-type stochastic calculus for anticipating integral processes, Bernoulli 10 (2004) 313-325.

[15] H. Triebel, Interpolation Theory, Function Spaces, Differential Operators, North-Holland, Amsterdam, 1978.

[16] Y. Xiao, T. Zhang, Local times of fractional Brownian sheets, Probab. Theory Related Fields 124 (2002) $204-226$. 